\title{
Analysis of Travel Mode and Departure Time Choice for Urban Shopping Trips
}

\author{
Chandra R. Bhat \\ Department of Civil Engineering \\ University of Texas at Austin
}

\begin{abstract}
Travel mode and departure time choice of urban trips are important determinants of urban travel demand and the temporal distribution of this demand. While mode choice has received substantial attention in travel demand modeling, relatively little attention has been directed toward departure time choice. Further, the work trip has been the focus of most earlier mode choice research, and almost all earlier departure time choice research. The current paper examines the joint nature of mode and departure time choice for urban shopping trips. The model formulation in the paper adopts a nested structure with mode choice at the higher level of the hierarchy and departure time choice at the lower level. A multinomial logit (MNL) form is used for modeling mode choice and an ordered generalized extreme value (OGEV) form, which recognizes the natural temporal ordering of the departure time alternatives, is adopted for departure time choice. The proposed MNL-OGEV model is applied to data obtained from the 1990 San Francisco Bay area travel survey and is found to perform better than the MNL and nested logit models. In addition, the results indicate that the MNL and nested logit models lead to biased level-of-service estimates and to inappropriate policy evaluations of transportation control measures in the current empirical context.
\end{abstract}

Keywords: generalized extreme-value model, nonwork trips, congestion pricing, peak period pricing, traffic congestion. 


\section{INTRODUCTION}

Mode choice and departure time choice are important components of a traveler's decision regarding trip-making. At an aggregate level, mode and departure time have a direct bearing on the number and temporal pattern of vehicle trips on urban roadways. Consequently, understanding the factors that affect travelers' mode and departure time choice is a necessary pre-requisite to examining the potential effectiveness of policy measures aimed at alleviating traffic congestion and reducing mobile-source emissions. In the travel demand literature, trip mode choice has received considerable attention. However, departure time choice has received relatively little attention. The limited research on departure time choice is reflected in the state-of-the-practice in travel choice modeling; local, state and federal transportation agencies do not have an explicit component to accommodate departure time choice in their travel model systems. These agencies apply simplistic, aggregatelevel, factors to apportion total daily travel demand among each of several time periods (for example, see Chang and Plager, 1994; p. 10).

Ignoring the time-of-day dimension of travel and applying factors that remain unchanged in the modeling process is inadequate for a number of reasons. First, the 1990 Clean Air Act Amendments (CAAAs) require travel demand models to provide accurate estimates of the number

of new vehicle trips during different times of the day. One can obtain such accurate estimates only by explicitly modeling the departure time of trips. Second, from a forecasting perspective, the application of static time-of-day factors does not consider the potential shifts in trip departure times due to non-uniform (across time-of-day) changes in network level-of-service between the estimation and forecast periods. This is likely to lead to inaccurate future year highway assignments by time of day. Third, from a policy standpoint, travel demand models have to be able to evaluate a variety of transportation control measures (TCMs) such as peak-period pricing, congestion-pricing, and ridesharing or transit-use incentives (see Stopher, 1993 and Weiner and Ducca, 1996). Many of these TCMs will not only have an impact on travel mode, but will also affect departure time choice.

Previous research on trip mode choice, and also the limited research on trip departure time choice, has primarily focused on the work trip (see Bhat, 1997a, Horowitz, 1993, Ben-Akiva and Lerman, 1985, Swait and Ben-Akiva, 1987, Fisher and Nagin, 1981, and Train, 1980 for work mode choice modeling; Abkowitz, 1981, Mannering, 1989, Chin, 1990, Hendrickson and Plank, 1984, Mahmassani and Jou, 1996, and Small, 1982 for work departure time choice modeling; and Chu, 1995 for the only study known to the author that models both work mode choice and departure time 
choice jointly). Nonwork travel, however, accounts for about three-fourths of the total trips in urban areas and projections suggest that this proportion is only likely to increase as suburbanization and lifestyle changes impact individuals' travel behavior (for a detailed discussion, see Lockwood and Demetsky, 1994).

The (growing) contribution of nonwork trips to urban traffic congestion and mobile source emissions, combined with the limited attention directed toward modeling such trips, forms the basis for our examination of nonwork trip mode and departure time choice. We direct our attention on the home-based shopping (HBS) purpose (within the broad category of nonwork trips) because the HBS purpose constitutes a major fraction of total nonwork trips.

The departure time choice for shopping trips is represented by several temporally contiguous discrete time periods such as AM peak (6AM-9AM), AM mid-day (9AM-12Noon), PM mid-day (12Noon-3PM), PM peak (3PM-6PM), and other (6PM-6AM). We use such a discrete time representation (rather than a continuous time representation) for two reasons. First, individuals generally have considerable departure time flexibility for shopping trips and are likely to choose among broad time periods rather than choose to pursue shopping at a precise continuous point in time (this is unlike for work activity where the work start time may restrict departure from home to a narrow window within which it might be most appropriate to consider time as being continuous). Second, an important reason for the current modeling effort is to better evaluate the effect of policy measures (such as peak period pricing) which are implemented during selective periods of the day and need to be reflected in models as time period-varying travel costs (or times). Accommodating such time-varying travel level-of-service measures is not straightforward in a continuous time model.

The paper formulates a joint model of mode and departure time choice using a nesting structure in which mode choice is modeled at the higher level of the hierarchy and departure time choice is modeled at the lower level. The alternative nesting structure in which departure time choice is at the higher level and mode choice at the lower level was found to be inconsistent with global utility maximization (the logsum parameter exceeded one). Intuitively, the nesting structure adopted in our paper implies that individuals are more likely to shift departure times than change travel modes in response to policies such as congestion pricing. The alternative nesting structure would imply that individuals are more likely to shift travel modes than departure times. In the context of 
shopping trips, there is generally substantial flexibility in departure time choice and hence it appears reasonable that travelers should be more likely to shift departure times than shift modes.

The nesting structure discussed above allows the joint choice alternatives to share unobserved attributes in the mode choice dimension, resulting in an increased sensitivity among time-of-day alternatives of the same mode relative to the time-of-day alternatives across modes. In addition to the correlation in departure time alternatives sharing the same mode, we also accommodate the natural ordering among the departure time alternatives along the time dimension by allowing the unobserved random utility components for adjacent departure time alternatives to be correlated within each mode. This generates an increased degree of sensitivity between adjacent departure time alternatives sharing the same mode. Our model uses the multinomial logit (MNL) formulation for the higher-level mode choice decision and the standard ordered generalized extremevalue (OGEV) formulation (see Small, 1987) for the lower-level departure time choice decision (we will refer to this joint model as the MNL-OGEV model). ${ }^{1}$

The rest of this paper is structured as follows. The next section develops the structure and presents the estimation procedure for the MNL-OGEV model. Section 3 discusses the data and the empirical results. This empirical section includes an empirical comparison of the performance of the MNL-OGEV model, the nested logit model, and the multinomial logit model. The final section provides a summary of the research findings.

\section{MODEL STRUCTURE AND ESTIMATION}

Consider a single traveler choosing among $I$ modal alternatives $(i=1,2, \ldots I)$ and $J$ departure times $(j$ $=1,2, \ldots J)$ for a shopping trip. Assume the following $G$ function within the class of Generalized Extreme Value (GEV) models proposed by McFadden (1978):

$$
G\left(y_{11}, y_{12}, \ldots y_{1 J}, y_{21}, y_{22}, \ldots y_{2 J}, \ldots y_{I 1}, \ldots y_{I 2}, \ldots y_{I J}\right)=\sum_{i=1}^{I}\left[\sum_{r=1}^{J+1}\left\{\frac{1}{2} y_{i r-1}^{1 / \rho_{p}}+\frac{1}{2} y_{i r}^{1 / \rho_{p}}\right\}^{\rho_{p} / \rho_{b}}\right]^{\rho_{b}}
$$

\footnotetext{
${ }^{1}$ The author was not aware of any earlier formulation of the MNL-OGEV model at the time of the writing of this paper. But, in response to the paper, Kenneth Small at the University of California, Irvine informed the author that the MNL-OGEV model form was written down in Small (1994). However, Small acknowledged that the current paper does much more in laying out the properties of the model and is the first known (to him) application of the model.
} 
where $0<\rho_{p} \leq \rho_{b} \leq 1, y_{i j} \geq 0$ for all $i, j$, and $y_{i 0}=y_{i J+1}=0$ for all $i$ by convention. Then, it is easy to verify that $G$ is non-negative, homogenous of degree one, tending toward $+\infty$ when any argument tends toward $+\infty$, and whose $n$th cross-partial derivatives are nonnegative for odd $n$ and nonpositive for even $n$ because $0<\rho_{p} \leq \rho_{b} \leq 1$. Thus, the following function represents a cumulative extreme-value distribution:

$$
F\left(\varepsilon_{11}, \varepsilon_{12}, \ldots \varepsilon_{1 J}, \varepsilon_{21}, \varepsilon_{22}, \ldots \varepsilon_{2 J}, \ldots \varepsilon_{I 1}, \varepsilon_{I 2}, \ldots \varepsilon_{I J}\right)=\exp \left\{-\sum_{i=1}^{I}\left[\sum_{r=1}^{J+1}\left\{\frac{1}{2} e^{-\varepsilon_{i r-1} / \rho_{p}}+\frac{1}{2} e^{-\varepsilon_{i r} / \rho_{p}}\right\}^{\rho_{p} / \rho_{b}}\right]^{\rho_{b}}\right\}
$$

Let $\varepsilon_{i j}$ in equation (2) represent the random element of utility for mode $i$ and departure time $j$. The marginal cumulative distribution function (CDF) of each stochastic element $\varepsilon_{i j}$ is univariate extreme-value as follows:

$$
F\left(\varepsilon_{i j}\right)=\exp \left[-\left\{2^{\rho_{b}-\rho_{p}}\right\} e^{-\varepsilon_{i j}}\right]
$$

The bivariate marginal CDF for two stochastic elements of different modes is the product of the corresponding univariate marginal CDF's; that is, the random elements of different modes are independent. The bivariate marginal CDF for two departure time alternatives sharing the same mode, but which are not adjacent in terms of departure time, is given by:

$$
H\left(\varepsilon_{i j}, \varepsilon_{i k}\right)=\exp \left(-\left\{2^{\rho_{b}-\rho_{p}}\right\}\left[e^{-\varepsilon_{i j} / \rho_{b}}+e^{-\varepsilon_{i k} / \rho_{b}}\right]^{\rho_{b}}\right), \text { for }|k-j|>1
$$

The above CDF generates a correlation between the utilities of two non-adjacent departure times within a mode that is the same as in a nested logit model; specifically,

$$
\operatorname{Cor}\left(\varepsilon_{i j}, \varepsilon_{i k}\right)=1-\rho_{b}^{2} \text { for }|j-k|>1
$$

Finally, the bivariate marginal CDF for two adjacent departure time elements sharing the same mode is as follows:

$$
H\left(\varepsilon_{i j}, \varepsilon_{i k}\right)=\exp \left(-\left\{2^{-\rho_{p}}\right\}\left[e^{-\varepsilon_{i j} / \rho_{b}}+e^{-\varepsilon_{i k} / \rho_{b}}+\left\{e^{-\varepsilon_{i j} / \rho_{p}}+e^{-\varepsilon_{i k} / \rho_{p}}\right\}^{\rho_{b} / \rho_{p}}\right] \rho_{b}\right), \text { for }|k-j|>1
$$


If $\rho_{p}=\rho_{b}$, then the expressions in equations (4) and (6) become identical and the model collapses to the familiar two-level nested logit model; thus, there is no covariance between the unobserved random utility elements of adjacent departure times conditional on mode choice. In addition, if $\rho_{p}=\rho_{b}=1$, then the model reduces to the MNL. In the general case, $0<\rho_{p}<\rho_{b}<1$, the correlation between adjacent departure time alternatives sharing the same mode cannot be written in closed form. However, the extent to which $\rho_{p}$ is lesser than $\rho_{b}$ can be interpreted as an index of the increased similarity between adjacent departure time alternatives relative to non-adjacent departure time alternatives. The actual correlation can be computed using numerical integration by noting that the marginal bivariate probability density function associated with the $\mathrm{CDF}$ in (6) is:

$$
\begin{aligned}
f\left(\varepsilon_{i j}, \varepsilon_{i k}\right)= & \left(2^{-\rho_{p}}\right) H\left(\varepsilon_{i j}, \varepsilon_{i k}\right) B_{i j k}^{\rho_{b}-2}\left[D_{i j k}\left(2^{-\rho_{p}} B_{i j k}^{\rho_{b}}+\left\{\frac{1-\rho_{b}}{\rho_{b}}\right\}\right)\right. \\
& \left.+B_{i j k}\left\{\frac{1}{\rho_{p}}-\frac{1}{\rho_{b}}\right\} e^{-\left(\varepsilon_{i j}+\varepsilon_{i k}\right) / \rho_{p}}\left(e^{-\varepsilon_{i j} / \rho_{p}}+e^{-\varepsilon_{i k} / \rho_{p}}\right)^{\frac{\rho_{p}}{\rho_{b}}-2}\right]
\end{aligned}
$$

where:

$$
\begin{aligned}
& B_{i j k}=e^{-\varepsilon_{i j} / \rho_{b}}+e^{-\varepsilon_{i k} / \rho_{b}}+C_{i j k}^{\rho_{p} / \rho_{b}}, C_{i j k}=\left(e^{-\varepsilon_{i j} / \rho_{p}}+e^{-\varepsilon_{i k} / \rho_{p}}\right), \text { and } \\
& D_{i j k}=\left(e^{-\varepsilon_{i j} / \rho_{b}}+e^{-\varepsilon_{i j} / \rho_{p}} C_{i j k}^{\left(\rho_{p} / \rho_{b}\right)-1}\right)\left(e^{-\varepsilon_{i k} / \rho_{b}}+e^{-\varepsilon_{i k} / \rho_{p}} C_{i j k}^{\left(\rho_{p} / \rho_{b}\right)-1}\right) .
\end{aligned}
$$

Table 1 presents the correlations for different combinations of $\rho_{p}$ and $\rho_{b}$. The diagonal entries in the matrix correspond to the correlations in a nested logit model $\left(\rho_{p}=\rho_{b}\right)$; equivalently, they correspond to the correlation between two non-adjacent departure time alternatives of the same mode in the MNL-OGEV model. As $\rho_{b}$ decreases, the correlation among departure time alternatives of the same mode (diagonal entries in Table 1) increases. In addition, as $\rho_{p}$ decreases, the correlation among adjacent departure time alternatives sharing the mode increases over and beyond the correlation between non-adjacent departure time alternatives. The correlation pattern is very flat between $\rho_{p}=0.2$ and $\rho_{p}=0$ and so Table 1 does not show the entries for $\rho_{p}<0.2$ (for each $\rho_{b}$ value, the entries for $\rho_{p}=0.2$ may be considered as the maximum possible correlation that can be generated between adjacent departure time alternatives of the same mode). 
To obtain the probability of choice for each mode-departure time alternative in the MNLOGEV model, consider a utility maximizing decision process where the utility of each modedeparture time alternative $\left(U_{i j}\right)$ is written in the usual form as the sum of a deterministic component $\left(V_{i j}\right)$ and a random component $\left(\varepsilon_{i j}\right)$. We assume a linear-in-parameters functional form so that $V_{i j}=\beta^{\prime} x_{i j}$. If the random components follow the CDF in (1), then, by the GEV postulate, the probability of choosing the ijth mode-departure time alternative is:

$$
P_{i j}=P_{j \mid i} \times P_{i}=\frac{e^{V_{i j} / \rho_{p}}\left\{\left(e^{V_{i j}-1 / \rho_{p}}+e^{V_{i j} / \rho_{p}}\right)^{\frac{\rho_{p}}{\rho_{b}}-1}+\left(e^{V_{i j} / \rho_{p}}+e^{V_{i j+1} / \rho_{p}}\right)^{\frac{\rho_{p}}{\rho_{b}}-1}\right\}}{\sum_{r=1}^{J+1}\left\{e^{V_{i r-1} / \rho_{p}}+e^{V_{i r} / \rho_{p}}\right\} \rho_{p} / \rho_{b}} \times \frac{e^{\rho_{b} W_{i}}}{\sum_{i^{\prime}=1}^{I} e^{\rho_{b} W_{i^{\prime}}}}
$$

where

$W_{i}=\ln \sum_{r=1}^{J+1}\left\{e^{V_{i r-1} / \rho_{p}}+e^{V_{i r} / \rho_{p}}\right\}^{\rho_{p} / \rho_{b}}$

To write the disaggregate self- and cross-elasticities of the joint model, we define the following two quantities:

$L_{i j}=\left\{e^{V_{i j}-1 / \rho_{p}}+e^{V_{i j} / \rho_{p}}\right\}^{\frac{\rho_{p}}{\rho_{b}}-2}$ and $M_{i j}=\left\{e^{V_{i j}-1 / \rho_{p}}+e^{V_{i j} / \rho_{p}}\right\}^{\frac{\rho_{p}}{\rho_{b}}-1}$

Then the disaggregate self- and cross-elasticities with respect to the lth level-of-service variable are as follows:

$$
\begin{aligned}
& \eta_{x_{l i j}}^{P_{i j}}=\left[\frac{1}{\rho_{p}}-\left(\frac{1-\rho_{b}}{\rho_{b}}\right) P_{j \mid i}-\left(\frac{1}{\rho_{p}}-\frac{1}{\rho_{b}}\right)\left(\frac{L_{i j}+L_{i j+1}}{M_{i j}+M_{i j+1}}\right) e^{V_{i j} / \rho_{p}}-P_{i j}\right] \beta_{l} x_{l i j} \\
& \eta_{x_{l i j}}^{P_{s k}}=-P_{i j} \beta_{l} x_{l i j} \text { for } s \neq i \\
& \eta_{x_{l i j}}^{P_{i k}}=-\left[\left(\frac{1-\rho_{b}}{\rho_{b}}\right) P_{j \mid i}+P_{i j}\right] \beta_{l} x_{l i j} \text { for }|k-j|>1
\end{aligned}
$$


$\eta_{x_{l i j}}^{P_{i k}}=\left[\left(\frac{1-\rho_{b}}{\rho_{b}}\right) P_{j \mid i}+P_{i j}+\left(\frac{1}{\rho_{p}}-\frac{1}{\rho_{b}}\right) \frac{\left(e^{V_{i j} / \rho_{p}}+e^{V_{i k} / \rho_{p}}\right)^{\rho_{p}}-2}{M_{i k}+M_{i k+1}} e^{V_{i j} / \rho_{p}}\right] \beta_{l} X_{l i j}$ for $|k-j|=1$

The cross-elasticity between mode-departure time alternatives that do not share the same mode (equation 11) is the same as that in the MNL. The structure of the cross-elasticity expression between two non-adjacent departure time alternatives within a mode (equation 12) is the same as that in the nested logit model. However, the cross-elasticity between two adjacent departure time alternatives which share the same mode is higher than the cross-elasticity between non-adjacent departure time alternatives because of the presence of the additional third term inside the square parentheses in equation (13). The presence of this third term in equation (13) also allows asymmetry in the draw between the two departure time alternatives $(k=j-1$ and $k=j+1)$ which are adjacent to the departure time alternative $(j)$ whose level-of-service is changed. To examine the nature of this response asymmetry, consider first the case when $\exp \left(V_{i j} / \rho_{p}\right)=\exp \left(V_{i q} / \rho_{p}\right)=W$, where we are using the index $q$ to label the departure time alternative adjacent to alternative $k$ on the other side of alternative $j$. Then, the third term inside the square parentheses in equation (13) is proportional to $\left[W+\exp \left(V_{i k} / \rho_{p}\right)\right]^{-1}$. Thus, generally speaking, the draw from an attractive adjacent departure time alternative $k$ is smaller than that from a relatively unattractive adjacent departure time alternative.

Aggregate own- and cross-elasticities, two measures of policy interest in terms of congestion-alleviation strategies, can be computed in a straight-forward manner from the disaggregate elasticities in equations (10) through (13) (see Ben-Akiva and Lerman, 1985, p. 113).

The estimation of the model specified by equation (8) can be achieved using maximumlikelihood techniques. One can use either the full-information technique or the limited-information technique. In the limited-information technique, the OGEV model of departure time choice is first estimated conditional on mode choice and then the marginal mode choice model is estimated using an estimate of $W_{i}$ constructed from the departure time model. However, the limited-information technique is inefficient and the expressions to obtain the correct asymptotic variance-covariance matrix of the parameters are tedious. Hence, we use the full-information procedure in our estimation. Maximization of the log-likelihood function is achieved using the GAUSS matrix programming language. The analytic gradients of the log-likelihood function with respect to the parameters are coded. The parameter estimates from the nested logit model (in which $\rho_{b}=\rho_{p}$ ) are used as the start 
parameters for the likelihood iterations. Though the likelihood function for estimation of the parameters in the MNL-OGEV model is not globally concave, we found the function to be wellbehaved and the iterations reached convergence in a stable fashion. In the estimations, we tried different starting points to test for more than one optima, but the iterations always converged to the same point.

\section{APPLICATION}

\subsection{Data}

The data for the study are drawn from the San Francisco Bay Area Household Travel Survey conducted by the Metropolitan Transportation Commission (MTC) in the Spring and Fall of 1990 (see White and Company, Inc., 1991 for details of survey sampling and administration procedures). This survey included a single-weekday travel diary of households, and it is this single-day sample that is used here. In addition to the travel diary, detailed individual and household sociodemographic information was also collected in the survey.

The modal alternatives include drive alone, shared-ride, and transit. The time-of-day choice is represented by five time-periods: AM peak (6AM-9AM), AM offpeak (9AM-12Noon), PM offpeak (12Noon-3PM), PM peak (3PM-6PM), and Evening (6PM-12 midnight). We also considered a sixth time period from 12 midnight to 6 am in the morning, but there were very few

shopping trips made in this period. Hence this period was excluded from the analysis. For some individual trips, modal availability may be a function of time-of-day (for example, transit mode is available only during the AM and PM peak periods) and this is accommodated by defining the feasible joint choice alternatives for each individual shopping trip.

Level of service data were generated for each zonal pair in the study area and by four time periods: am peak, mid-day, pm peak, and evening. The Metropolitan Transportation Commission in Oakland provided zone-to-zone level-of-service data by travel mode for two time periods of the day: AM peak and mid-day. We applied mode-specific factors to the AM peak and mid-day level-ofservice data to obtain the level-of-service measures for the other time periods of the day. The factors were developed based on information extracted from the household travel survey. For a detailed discussion of the procedure, see Bhat (1997b). The impedance data were appropriately appended to the home-based trips based on the origin-destination of trips. 
The sample used in this paper comprises 4516 home-based person-shopping trips obtained from the overall single-day travel diary sample. The mode choice shares in the sample are as follows: drive alone (61.3\%), shared-ride (37.6\%) and transit (1.0\%). The departure time distribution of home-based shopping trips is as follows: AM peak (3.7\%), AM offpeak (21.2\%), PM offpeak (29.8\%), PM peak (20.7\%), and evening (24.6\%).

\subsection{Model Variables}

Four sets of variables were used in the model specification: a) alternative specific constants (since there are $I=3$ modal alternatives and $J=5$ departure time alternatives, there are $I * J=15$ joint alternatives in the universal choice set and so we included 14 alternative specific constants), b) individual/household sociodemographics, c) trip destination attributes, and d) level-of-service variables. We arrived at the final specification based on a systematic process of eliminating variables found to be statistically insignificant in previous specifications. The sociodemographic variables influencing modal choice in the final specification included employment status (whether employed or not), a non-caucasian race indicator, age, sex, an elderly flag indicator (whether above 65 years or not), number of cars in individual's household, and a flag variable indicating presence of children (less than or equal to 16 years) in the individual's household. The sociodemographic variables affecting departure time choice included all the variables mentioned above except number of cars and presence of children. There was no statistically significant effect of the sociodemographics on the joint mode-departure time choice after accommodating the unidimensional effects of the variables on mode and departure time choice. The trip destination attributes (affecting the modal and time-of-day dimensions separately) included a San Francisco downtown destination indicator that identified whether a trip terminated in the San Francisco downtown area, and a Central Business District (CBD) destination flag that indicated whether a trip terminated in a CBD. ${ }^{2}$ Three level-ofservice variables were used in the current analysis: travel cost, total travel time, and out-of-vehicle travel time over trip distance.

\footnotetext{
2 The CBD districts include the San Francisco superdistricts (except the downtown superdistrict which has an extremely high employment density and is identified separately) and the superdistricts of San Jose and Oakland. The superdistrict classification is based on a 34 system categorization developed by the Metropolitan Transportation Commission.
} 


\subsection{Empirical Results}

We estimated three different models of mode-departure time choice: the multinomial logit (MNL) model, the nested logit (NL) model with departure time alternatives within each mode specified to share common unobserved random utility attributes (as indicated earlier, the alternative nesting structure was found to be inappropriate), and the MNL-OGEV model proposed in this paper.

The level-of-service parameter estimates, implied money values of time, correlation parameters, and data fit measures from the models are presented in Table 2 (other parameter estimates are presented in section 3.5). The signs of the level-of-service parameters are consistent with a priori expectations in all the models. Also, as expected, travelers are more sensitive to out-ofvehicle travel time than in-vehicle travel time. The implied money values of in-vehicle and out-ofvehicle travel times across the three specifications reveals a progressively decreasing sensitivity to time (relative to cost) as we move from the MNL model to the MNL-OGEV model.

The differences in sensitivity among the MNL, NL, and MNL-OGEV models suggest the need to apply formal statistical tests to determine the structure that is most consistent with the data. The three models can be evaluated formally by using conventional likelihood ratio tests. A statistical comparison of the multinomial logit model with the nested logit model leads to the rejection of the multinomial logit. A further likelihood ratio test between the nested logit and MNL-OGEV models results in the clear rejection of the hypothesis that adjacent departure time alternatives sharing the same mode do not have a heightened degree of sensitivity compared to non-adjacent departure time alternatives (the likelihood ratio test value is 10.08 which is larger than the chi-squared statistic with 1 degree of freedom at any reasonable level of significance). Thus, both the multinomial logit and nested logit models are mis-specified. This can also be observed by noting the asymptotic t-statistics on $\rho_{p}$ and $\rho_{b}$ in the MNL-OGEV model; $\rho_{b}$ is significantly smaller than one and $\rho_{p}$ is significantly smaller than $\rho_{b}$.

The correlation structure implied by the three models is presented in Table 3. The MNL model assumes zero correlation between the random utility components of any pair (and all pairs) of mode-departure time alternatives. The nested logit model shows an identical degree of correlation among departure time alternatives sharing the same mode. The MNL-OGEV model indicates a higher degree of correlation between adjacent departure time alternatives compared to the correlation between non-adjacent departure times within a mode. It appears that the correlation 
between adjacent alternatives because of the ordering effect in departure time choice is being absorbed into the correlation among alternatives sharing the same mode in the nested logit model, giving a mis-perception of a high correlation among all pairs of departure time alternatives of the same mode in the nested logit.

The different correlation structures among the MNL, nested logit and MNL-OGEV models imply different patterns of competition among the joint mode-departure time alternatives. Table 4 presents the disaggregate self- and cross-elasticities (for the first shopping person-trip in the sample) in response to peak period pricing implemented in the PM peak (i.e., a cost increase in the "drive alone-PM peak" alternative). The MNL model exhibits the familiar Independent from Irrelevant Alternatives (IIA) property. The cross-elasticities from the nested logit are higher for the joint alternatives which include the drive alone alternative (we will refer to such alternatives as drive alone joint choice alternatives) relative to other non-drive alone joint choice alternatives, but the IIA assumption is maintained among the drive alone joint choice alternatives. Finally, the crosselasticities from the MNL-OGEV model indicate higher cross-elasticities for the adjacent DA-PM offpeak and DA-evening alternatives compared to the non-adjacent DA-AM peak and DA-AM offpeak alternatives. The higher cross-elasticity for the adjacent DA-PM offpeak alternative relative to the adjacent DA-Evening alternative is because the DA-evening alternative is more attractive for the person-trip under consideration.

\subsection{Substantive Policy Implications}

The disaggregate elasticities provide an intuitive idea of the structure of each model and present the nature of inter-alternative competition at the individual-level. We now turn to the aggregate self- and cross-elasticities to examine the substantive implications for the level-of-service variables. To limit the discussion, we focus only on the travel cost elasticities for the drive alone and transit joint choice alternatives in response to a congestion pricing policy implemented in the PM peak.

Table 5 provides the cost elasticities obtained from the MNL-OGEV model and the nested logit model (we do not show the cost elasticities from the MNL for ease in exposition). The aggregate cost cross-elasticities are about the same for the transit joint choice alternatives in both the NL and MNL-OGEV models. However, the cross-elasticities from the two models are quite different for the drive alone joint choice alternatives. Specifically, the MNL-OGEV model shows a much higher displacement to the adjacent DA-PM offpeak and DA-evening alternatives (the 
elasticities are about $80 \%$ higher in the MNL-OGEV model relative to the NL model). The MNLOGEV model also estimates a lower displacement to the DA-AM peak and DA-AM offpeak alternatives. Finally, the MNL-OGEV model estimates a larger reduction in drive alone mode share in the PM peak in response to peak-period pricing relative to the NL (as evidenced in the selfelasticity values).

\subsection{Detailed MNL-OGEV Model Results}

In this section, we present and discuss the parameter estimation results from the MNL-OGEV model (see Table 6). We do not present the 15 alternative-specific constant values due to space constraints. We also do not discuss the effect of level-of-service variables or the correlation parameters, since these have been presented earlier in Table 2.

Among the socio-demographic variables, we observe that employed individuals tend to shop primarily during the evening period. Employed individuals are particularly unlikely to shop during the AM offpeak (or midday) and PM offpeak (or midday) periods since they would be at work during these times. The effect of employment on mode choice indicates that employed individuals are more likely to use the drive alone mode for shopping than unemployed individuals. Age has a negative effect on making shopping trips in the evening. This effect is particularly strong for individuals over the age of 65 years. Older people are more likely to use the drive alone mode to

shop, but this trend reverses sharply for individuals over 65 years of age. Women tend to shop during the AM/PM offpeak (mid-day) and PM peak periods than in the AM peak and evening periods. This may be a reflection of household responsibilities shouldered by women, which makes it difficult for them to pursue out-of-home activities in the early and late parts of the day. The effect of sex on mode use suggests that women are more predisposed toward transit-use than men. The effects of other socio-demographic variables on mode choice are also reasonable. The strong positive effect of presence of children on use of the shared-ride mode is simply a reflection of adults traveling with their children to shop.

The impact of the trip destination attributes on departure time choice shows that individuals are unlikely to shop in the evening period (relative to other time periods) if their shopping destination is in the San Francisco downtown area or in the other Central Business districts (CBDs) of the Bay area. The effect of these trip destination attributes on mode choice indicates that individuals who shop in the San Francisco downtown area or other CBDs are highly likely to use the 
transit mode. This is to be expected because of the high traffic congestion in these areas and also since these corridors are likely to be well served by transit due to their high land-use densities.

\section{CONCLUSIONS}

In this paper, we have formulated and estimated a joint model of mode choice and departure time choice which recognizes the natural temporal ordering among the departure time alternatives. The model allows an increased degree of choice sensitivity between adjacent departure time alternatives sharing the same mode. The model structure uses a multinomial logit (MNL) form for the higherlevel mode choice decision and Small's (1987) standard ordered generalized extreme-value (OGEV) form for the lower-level departure time choice decision. The model is parsimonious (it has only one additional parameter over the nested logit model) and at the same time allows a more flexible correlation structure among alternatives than does the nested logit model. It has closed-form expressions for the choice probabilities and is therefore much easier to estimate than a multinomial probit model. This is particularly important when the number of alternatives is very large, as is likely to be the case in multi-dimensional contexts.

The empirical analysis of the paper applied the multinomial logit, the nested logit, and the proposed MNL-OGEV formulations to the estimation of mode-departure time choice for homebased shopping trips using data drawn from the 1990 Bay area household travel survey. The results indicate that the nested logit model outperforms the MNL model in terms of data fit and further that the MNL-OGEV model outperforms the nested logit model on the same criterion. We also find that failure to accommodate the correlation in unobserved utility components of departure time alternatives sharing the same mode, and the additional correlation between each pair of adjacent departure time alternatives sharing the same mode, leads to biased estimates of the effects of levelof-service variables. For example, as illustrated earlier, the MNL and NL models underestimate the reduction in PM peak period congestion due to implementation of congestion pricing in the PM peak. The MNL and NL models also underestimate the displacements of drive alone mode share to the adjacent PM offpeak and PM evening time periods. In summary, the MNL and NL models can lead to inappropriate evaluations of transportation control measures and, consequently, mis-informed policy actions. 


\section{ACKNOWLEDGEMENTS}

This research was supported by National Science Foundation grants DMS 9208758 and DMS 9313013 to the National Institute of Statistical Sciences (NISS). Mr. Charles Purvis of the Metropolitan Transportation Commission in Oakland, California provided the 1990 Bay area household travel survey data and clarified several data-related issues. The author would like to thank Kenneth Small and two anonymous referees for valuable comments on an earlier version of the paper. 


\section{REFERENCES}

Abkowitz, M.D. (1981) An analysis of the commuter departure time decision, Transportation, 10, 283-297.

Ben-Akiva, Moshe and Steven R. Lerman (1985) Discrete Choice Analysis, MIT Press, Cambridge.

Bhat, C.R. (1997a) Incorporating observed and unobserved heterogeneity in urban work travel choice modeling, manuscript, Department of Civil Engineering, University of Texas, Austin.

Bhat, C.R. (1997b) Generation of zone-to-zone level-of-service data by time period of day for the Bay area, technical note, Department of Civil Engineering, University of Texas, Austin.

Chang, Y.B. and M. Plager (1994) Interim Regional Travel Demand Forecasting Model: Development of the 1993 Version, Memorandum, June 17.

Chin, A.T.H (1990) Influences on commuter trip departure time decisions in Singapore, Transportation Research, 24A, 321-333.

Chu, X., (1995) Alternative congestion pricing schedules, paper presented at the 79th American Economic Association Annual Meeting, Washington, D.C., January.

Fischer, G.W. and D. Nagin (1981) Random versus fixed coefficient quantal choice models, in Manski, C.F. and D. McFadden (eds.) Structural Analysis of Discrete Data with Econometric Applications, MIT Press, Cambridge, Massachusetts, 273-304.

Hendrickson, C. and E. Plank (1984) The flexibility of departure times for work trips, Transportation Research, 18A, 25-36.

Horowitz, J.L. (1993) Semiparametric estimation of a work trip mode choice model, Journal of Econometrics, 58, 49-70.

Lockwood, P.B. and M.J. Demetsky (1994) Nonwork travel - a study of changing behavior, presented at the 73rd Meeting of the Transportation Research Board, Washington, D.C.

Mahmassani, H.S. and R-C. Jou (1996) Bounded rationality in commuter decision dynamics: Incorporating trip chaining in departure time and route switching decisions, presented at the Conference on Theoretical Foundations of Travel Choice Modeling, Stockholm.

Mannering, F.L. (1989) Poisson analysis of commuter flexibility in changing routes and departure times, Transportation research, 23B, 53-60.

McFadden, D. (1978) Modelling the choice of residential location in A. Karlquist (ed.) Spatial Interaction Theory and Residential Location, North Holland, Amsterdam, 75-96.

Small, K.A. (1982) The scheduling of consumer activities: Work trips, American Economic Review, 72, 467-479.

Small, K.A. (1987) A discrete choice model for ordered alternatives, Econometrica, 55(2), 409424. 
Small, K.A. (1994) Approximate generalized extreme value models of discrete choice, Journal of Econometrics, 62, 351-382.

Stopher, P.R. (1993) Deficiencies of travel-forecasting methods relative to mobile emissions, Journal of Transportation Engineering, 119, 5, 723-741.

Swait, J. and M. Ben-Akiva (1987) Empirical test of a constrained choice discrete model: mode choice in Sao Paulo, Brazil, Transportation Research, 21B, 103-115.

Train, K. (1980) A structured logit model of auto ownership and work mode choice, Review of Economic Studies, 64, 357-370.

Weiner, E. and F. Ducca (1996). Upgrading Travel Demand Forecasting Capabilities: USDOT Travel Model Improvement Program, TR News, 186, 2-6.

White, E.H. and Company, Inc. (1991) 1990 Bay Area Travel Survey: Final Report, submitted to the Metropolitan Transportation Commission, Oakland, California. 


\section{List of Tables}

Table 1: Correlation between Stochastic Utilities of Adjacent Departure Time Alternatives of the Same Mode

Table 2: Level of Service Parameters, Implied Money values of Travel Time, Correlation Parameters, and Data Fit Measures

Table 3: Correlation Between Stochastic Utility Components of Mode-Departure Time Alternatives

Table 4: Disaggregate Travel Cost Elasticities in Response to a Cost Increase in the Drive Alone (DA) Mode during PM Peak

Table 5: Aggregate Cost Elasticities in Response to a Cost Increase in the Drive Alone (DA) Mode during PM Peak

Table 6: MNL-OGEV Model Results 
Table 1: Correlation between Stochastic Utilities of Adjacent Departure Time Alternatives of the Same Mode

\begin{tabular}{|c|c|c|c|c|c|c|c|c|c|}
\hline \multirow{2}{*}{$\rho_{p}$} & \multicolumn{9}{|c|}{$\rho_{b}$} \\
\hline & 1.0 & 0.9 & 0.8 & 0.7 & 0.6 & 0.5 & 0.4 & 0.3 & 0.2 \\
\hline 1.0 & 0.000 & & & & & & & & \\
\hline 0.9 & 0.094 & 0.190 & & & & & & & \\
\hline 0.8 & 0.175 & 0.270 & 0.360 & & & & & & \\
\hline 0.7 & 0.245 & 0.344 & 0.432 & 0.510 & & & & & \\
\hline 0.6 & 0.304 & 0.405 & 0.494 & 0.572 & 0.640 & & & & \\
\hline 0.5 & 0.353 & 0.454 & 0.545 & 0.625 & 0.693 & 0.750 & & & \\
\hline 0.4 & 0.392 & 0.494 & 0.585 & 0.666 & 0.735 & 0.794 & 0.840 & & \\
\hline 0.3 & 0.422 & 0.524 & 0.616 & 0.698 & 0.768 & 0.827 & 0.876 & 0.910 & \\
\hline 0.2 & 0.435 & 0.529 & 0.618 & 0.700 & 0.774 & 0.838 & 0.890 & 0.930 & 0.960 \\
\hline
\end{tabular}


Table 2: Level of Service Parameters, Implied Money values of Travel Time, Correlation Parameters, and Data Fit Measures

\begin{tabular}{|c|c|c|c|}
\hline Attributes/data fit measures & MNL model & NL model & MNL-OGEV model \\
\hline \multicolumn{4}{|l|}{ Level of service ${ }^{a}$} \\
\hline Travel cost & $-0.0061(-4.52)$ & $-0.0052(-2.82)$ & $-0.0050(-2.08)$ \\
\hline In-vehicle travel time & $-0.0274(-2.80)$ & $-0.0211(-2.32)$ & $-0.0161(-2.18)$ \\
\hline Out-of-vehicle time/distance & $-0.1078(-3.52)$ & $-0.0714(-2.03)$ & $-0.0506(-1.94)$ \\
\hline \multicolumn{4}{|l|}{ Implied money values of time $(\$ / \mathrm{hr})$} \\
\hline In-vehicle travel time & 2.70 & 2.43 & 1.93 \\
\hline Out-of-vehicle travel time & 5.40 & 4.53 & 3.47 \\
\hline LL at Convergence ${ }^{b}$ & -9123.18 & -9116.13 & -9111.09 \\
\hline \multicolumn{4}{|l|}{ Correlation parameters $^{a}$} \\
\hline$\rho_{p}$ & $1.000(-)^{c}$ & same as $\rho_{b}(-)^{c}$ & $0.445(3.89)^{c}$ \\
\hline$\rho_{b}$ & $1.000(-)^{d}$ & $0.719(3.36)^{d}$ & $0.812(2.20)^{d}$ \\
\hline
\end{tabular}

a The entries in the different columns correspond to the parameter values and their t-statistics (in parenthesis).

${ }^{b}$ The LL (log-likelihood) at equal shares is -12174.82 and the LL with only alternative specific constants and an IID error covariance matrix is -9891.90 .

${ }^{c} \rho_{p}$ is implicitly constrained to one in the MNL model; it is implicitly constrained to be equal to $\rho_{b}$ in the nested logit model. The asymptotic t-statistic for $\rho_{p}$ in the MNL-OGEV model is with respect to the value of $\rho_{b}$ (the standard error of the difference between $\rho_{b}$ and $\rho_{p}$ is obtained from the variance-covariance matrix of the estimated parameters).

${ }^{d} \rho_{b}$ is implicitly constrained to one in the model. The asymptotic t-statistics for $\rho_{b}$ in the nested and MNLOGEV models are with respect to a value of one. 
Table 3: Correlation Between Stochastic Utility Components of Mode-Departure Time Alternatives

\begin{tabular}{|l|c|c|c||}
\hline Correlation between joint choice alternatives & MNL & NL & MNL-OGEV \\
\hline of different modes & 0.000 & 0.000 & 0.000 \\
\hline sharing the same mode, but non-adjacent in departure time & 0.000 & 0.481 & 0.339 \\
\hline sharing the same mode and adjacent in departure time & 0.000 & 0.481 & 0.558 \\
\hline
\end{tabular}

Table 4: Disaggregate Travel Cost Elasticities in Response to a Cost Increase in the Drive Alone (DA) Mode during PM Peak

\begin{tabular}{|c|c|c|c|}
\hline Effect on Joint Choice Alternative & MNL model & Nested Logit model & MNL-OGEV model \\
\hline DA-AM peak & 0.055 & 0.071 & 0.060 \\
\hline DA-AM offpeak & 0.055 & 0.071 & 0.060 \\
\hline DA-PM offpeak & 0.055 & 0.071 & 0.122 \\
\hline DA-PM peak & -0.225 & -0.259 & -0.332 \\
\hline DA-evening & 0.055 & 0.071 & 0.106 \\
\hline All other joint alternatives & 0.055 & 0.046 & 0.046 \\
\hline
\end{tabular}


Table 5: Aggregate Cost Elasticities in Response to a Cost Increase in the Drive Alone (DA) Mode during PM Peak

\begin{tabular}{||l|c|c|}
\hline Effect on Joint Choice Alternative & NL model & MNL-OGEV model \\
\hline \hline Drive alone alternatives & & 0.035 \\
AM peak & 0.042 & 0.030 \\
AM offpeak & 0.037 & 0.069 \\
PM offpeak & 0.039 & -0.293 \\
PM peak & -0.234 & 0.092 \\
Evening & 0.051 & \\
\hline \hline Transit Alternatives & & 0.047 \\
AM peak & 0.049 & 0.032 \\
AM offpeak & 0.033 & 0.035 \\
PM offpeak & 0.036 & 0.046 \\
PM peak & 0.046 & 0.040 \\
Evening & 0.041 & \\
\hline
\end{tabular}


Table 6: MNL-OGEV Model Results

\begin{tabular}{|c|c|c|c|c|}
\hline \multirow{2}{*}{ Variables } & \multicolumn{2}{|c|}{$\begin{array}{c}\text { Departure Choice } \\
\text { Sub-Model }\end{array}$} & \multicolumn{2}{|c|}{$\begin{array}{l}\text { Mode Choice } \\
\text { Sub-Model }\end{array}$} \\
\hline & Parameter & t-statistic & Parameter & t-statistic \\
\hline \multicolumn{5}{|l|}{ Socio-demographic Attributes } \\
\hline \multicolumn{5}{|l|}{ Employment status } \\
\hline AM peak & -0.7171 & -2.13 & - & - \\
\hline AM offpeak & -1.2890 & -2.31 & - & - \\
\hline PM offpeak & -1.0375 & -2.33 & - & - \\
\hline PM peak & -0.3786 & -2.13 & - & - \\
\hline Drive alone & - & - & 0.4097 & 4.92 \\
\hline \multicolumn{5}{|l|}{ Age } \\
\hline Evening & -0.0158 & -2.25 & - & - \\
\hline Drive alone & - & - & 0.0203 & 6.30 \\
\hline \multicolumn{5}{|l|}{ Elderly (> 65 years) } \\
\hline Evening & -0.4256 & -2.11 & - & - \\
\hline Drive alone & - & - & -0.8798 & -6.87 \\
\hline \multicolumn{5}{|l|}{ Female } \\
\hline AM/PM offpeak, PM peak & 0.1174 & 1.92 & - & - \\
\hline Transit & - & - & 0.6082 & 1.71 \\
\hline Non-caucasian (specific to drive alone) & - & - & -0.2627 & -3.27 \\
\hline Presence of children (specific to shared ride) & - & - & 1.0635 & 8.82 \\
\hline Number of vehicles (specific to transit) & - & - & -1.5552 & -2.93 \\
\hline \multicolumn{5}{|l|}{ Trip Destination Attributes } \\
\hline \multicolumn{5}{|l|}{ San Francisco downtown } \\
\hline Evening & -0.9589 & -2.49 & - & - \\
\hline Transit & - & - & 4.1455 & 6.61 \\
\hline \multicolumn{5}{|l|}{ Other CBDs } \\
\hline Evening & -0.2234 & -1.90 & - & - \\
\hline Transit & - & - & 2.6633 & 5.59 \\
\hline
\end{tabular}

